\title{
Protein kinase CK2 subunits are positive regulators of AKT kinase
}

\author{
BARBARA GUERRA \\ Institute for Biochemistry and Molecular Biology, University of Southern Denmark, DK-5230 Odense, Denmark
}

Received September 20, 2005; Accepted November 11, 2005

\begin{abstract}
Protein kinase CK2 is a highly conserved serine/ threonine kinase ubiquitously distributed in all investigated eukaryotes and involved in several intracellular pathways which control, among others, cell cycle, proliferation, apoptosis and transformation. Similarly, the PI3K-AKT pathway deregulation has been shown to play an important role in tumor development and tumor response to anti-cancer treatment. In this study, it is shown that the specific inhibition of the PI3KAKT signaling pathway in combination with depletion of CK2 subunits by antisense oligodeoxynucleotides leads to an enhanced drug-induced apoptotic response. Moreover, in vitro as well as in vivo studies show that the individual CK2 subunits interact with AKT kinase and that the complex formation is not modulated by the phosphorylation status of AKT. Furthermore, the fact that the interaction between CK2 subunits and AKT enhances AKT kinase activity identifies a novel molecular mechanism that leads to modulation of AKT activation raising the possibility that $\mathrm{CK} 2$ and AKT might be implicated in common pathways that control cell proliferation and survival.
\end{abstract}

\section{Introduction}

Protein kinase CK2 is a serine/threonine kinase ubiquitously distributed and highly conserved among all the organisms investigated so far. It is composed of two catalytic subunits $\alpha$ and/or $\alpha^{\prime}$ and two regulatory subunits $B$. The level of CK2 and kinase activity are elevated in solid tumors as well as in highly proliferative tissues (reviewed in ref. 1). CK2 has been detected in virtually all cellular compartments and in association with specific structures e.g., the plasma membrane, the Golgi apparatus, ribosomes etc. (reviewed in ref. 2). Because of the broad spectrum of proteins interacting with either the CK2 holoenzyme, the CK2 catalytic subunit and the CK2 regulatory subunit or that are phosphorylated by this kinase, it may not be surprising that CK2 is implicated in several cellular

Correspondence to: Dr Barbara Guerra, Institute for Biochemistry and Molecular Biology, University of Southern Denmark, Campusvej 55, DK-5230 Odense, Denmark

E-mail: bag@bmb.sdu.dk

Key words: CK2, AKT1, protein-protein interaction, phosphorylation, apoptosis functions. CK2 is involved in cellular growth, cell cycle progression, cell transformation and apoptosis. The creation of transgenic mice carrying deregulated expression of CK2subunits has shown to be an effective way to study the cellular consequences of CK2 altered expression (3-7). Moreover, the effect of CK2 subunits depletion has also been extensively studied. The suppression of CK2 catalytic subunits expression achieved through the use of antisense oligodeoxynucleotides techniques and/or the utilization of specific CK2 inhibitors has provided strong evidence that CK2 promotes cell growth, negatively modulating the apoptotic response of cells exposed to compounds known to induce cell death (reviewed in ref. 1).

The phosphatidylinositol 3-kinase (PI3K)/AKT signal transduction pathway has been shown to be central in many intracellular processes such as the insulin-dependent metabolic cell response, cell survival, proliferation, angiogenesis and motility (reviewed in ref. 8). The stimulation of membrane receptors by growth factors such as insulin-like growth factor-1 (IGF-1) and platelet-derived growth factor (PDGF) activates PI3K allowing the localization of phosphatidylinositoldependent kinase 1 (PDK1) at the cell membrane via its pleckstrin homology $(\mathrm{PH})$ domain (reviewed in ref. 8). The concomitant translocation and subsequent binding of AKT kinase from the cytoplasm to the inner surface of the plasma membrane leads to PDK1-mediated AKT phosphorylation and activation. Mammalian AKT family members comprise three isoforms (AKT1, 2, 3) highly conserved throughout evolution but differently expressed at both the mRNA and protein levels (reviewed in ref. 9). In vitro and in vivo experiments have revealed that active AKT is a phosphoprotein and although Thr308 and Ser473 on AKT1 have been shown to be the primary regulatory phosphorylation sites, different research groups have identified a number of phosphorylation sites with putative regulatory significance (9). The search for substrates of AKT has led to the identification of several components of the apoptotic machinery, giving rise to the idea that $\mathrm{AKT}$ is a key element in the regulation of cell survival (10-13). The notion that AKT and, in general, the PI3K pathway are involved in the regulation of uncontrolled cell proliferation has been strengthened by the fact that the PI3K pathway is negatively regulated by PTEN, a dual-specificity lipid and protein phosphatase considered to be a tumor suppressor gene product (14). Many of the proteins that are targeted by AKT are not strictly substrates for AKT kinase but rather proteins that interact with this kinase modulating AKT kinase function and activity (reviewed in ref. 15). Interestingly, several AKT-interacting 
proteins are protein kinases. PDK1 kinase, which targets AKT for phosphorylation at the cell membrane is also an AKT binding protein (16). In keratinocytes, keratin K10 sequesters PKC $\zeta$ in complex with AKT to the keratin cytoskeleton maintaining both kinases in an inactive state inducing cell cycle arrest (17).

Although PDK1-dependent activation of AKT has been extensively studied, the aim of the present study was to investigate whether additional pathways might control the activity of AKT in cells. Moreover, the fact that CK2 and AKT play important roles in cell survival and are both negative regulators of programmed cell death, suggested a possible link between these two protein kinases.

Here, it is reported that inhibition of the PI3K pathway induced by wortmannin, a fungal metabolite, enhances the nocodazole-induced apoptotic response of cells depleted of CK2 subunits. Heterocomplex formation between AKT and CK2 subunits occurs in vitro and in vivo and enhances the kinase activity of AKT. The physical association of AKT with CK2 and the observation that CK2 subunits enhance the kinase activity of AKT raise the possibility that CK2 might act in concert with AKT in signaling pathways that promote proliferation and protect cells from apoptosis.

\section{Materials and methods}

Cell culture and treatments. The GV10.15 cell line stably expressing CK2ß-Myc was cultured in Dulbecco's modified Eagle's medium (DMEM, Gibco) in the presence of $10 \%$ fetal bovine serum (FBS), $460 \mu \mathrm{g} / \mathrm{ml} \mathrm{G} 418$ (Gibco) and with or without $1 \mu \mathrm{g} / \mathrm{ml}$ Tetracycline (Sigma). HeLa and Cos-1 cell lines were grown in DMEM supplemented with $10 \%$ FBS. K562 and HL-60 cell lines were cultured in Roswell Park Memorial Institute medium (RPMI, Gibco) supplemented with $10 \% \mathrm{FBS}$ and $2 \mathrm{mM} \mathrm{L-glutamine.} \mathrm{All} \mathrm{cell} \mathrm{lines} \mathrm{were} \mathrm{grown}$ at $37^{\circ} \mathrm{C}$ under a $5 \% \mathrm{CO}_{2}$ atmosphere. For the in vivo activation of AKT, cells were seeded and $24 \mathrm{~h}$ thereafter they were subjected to serum starvation for $24 \mathrm{~h}$ and then treated with $50 \mathrm{ng} / \mathrm{ml} \mathrm{IGF-1} \mathrm{(Calbiochem)} \mathrm{for} 15 \mathrm{~min}$. The transient overexpression of proteins indicated in the figure legends was performed with the corresponding plasmids described elsewhere (18) using FuGene 6 reagent (Roche) following the manufacturer's recommendations. In the case of AKT activation, cells were transfected $24 \mathrm{~h}$ prior cell starvation. Where indicated, cells were incubated with $100 \mathrm{nM}$ wortmannin (Sigma) $1 \mathrm{~h}$ prior to the induction of apoptosis by incubating HeLa cells with $400 \mathrm{ng} / \mathrm{ml}$ nocodazole (Calbiochem) for $24 \mathrm{~h}$.

The depletion of cells of CK2 catalytic subunits was achieved by the antisense oligodeoxynucleotide technique (ASO) as described previously (19). The additional silencing of CK2 $2 \beta$ expression was achieved with the antisense CK2 $\beta$ oligodeoxynucleotide sequence 5'-AGC TAC TCA TCT TTA TGT-3' (DNA Technology).

Antibodies. Proteins were detected using primary antibodies against the HA-tag (mouse monoclonal anti-HA antibody, HA.11 Covance), AKT (mouse monoclonal anti-AKT antibody, BD Transduction Laboratories), phospho-AKT(Thr308) (rabbit polyclonal anti-phospho-AKT antibody, Cell Signaling),
phospho-AKT(Ser473) (mouse monoclonal anti-phospho-AKT antibody, 587F11 Cell Signaling), myc-tag (mouse monoclonal anti-Myc antibody, 9E10 Santa Cruz), CK2 $\alpha$ (mouse monoclonal anti-CK2 $\alpha$ antibody, 1AD9 Calbiochem), CK2ß (mouse monoclonal anti-CK2ß antibody, 6D5 Calbiochem), actin (mouse monoclonal anti- $\beta$ actin antibody, Sigma). Proteinantibody complexes were visualized by a chemiluminescence detection system following the manufacturer's guidelines (CDP-Star, Applied Biosystems).

Cell extracts, immunoprecipitation, and Western blot analysis. Prior to harvesting, cells were washed twice in cold PBS, spun down and resuspended in lysis buffer $(50 \mathrm{mM}$ Tris/HCl $\mathrm{pH} 7.5,150 \mathrm{mM} \mathrm{NaCl}, 1 \%$ Triton X-100, 10\% glycerol, $1 \mathrm{mM}$ DTT, $1 \mathrm{mM} \mathrm{Na} \mathrm{VO}_{4}, 30 \mathrm{mM}$ B-glycerophosphate, $10 \mathrm{mM}$ $\mathrm{NaF}, 100 \mathrm{nM}$ okadaic acid) containing a protease inhibitor cocktail (Roche). After sonication, cells were centrifuged at $4^{\circ} \mathrm{C}$ for $30 \mathrm{~min}$ at $10000 \mathrm{x} \mathrm{g}$. Whole cell extracts were then subjected to SDS-PAGE, Western blot analysis, protein kinase assay or immunoprecipitation experiments. Immunoprecipitation experiments were performed essentially as described previously (20) using $500 \mu \mathrm{g}$ protein lysates and in the presence of either rabbit polyclonal anti-CK2ß serum, rabbit polyclonal anti-CK $2 \alpha$ serum (both antibodies were obtained immunizing rabbits against the full-length proteins) or rabbit polyclonal anti-AKT antibody (Upstate Biotechnology).

Preparation of various recombinant human proteins. Recombinant human CK2 $\alpha$ and CK2 holoenzyme were expressed and purified essentially as described elsewhere (21). Wildtype CK2ß was expressed in E. coli and purified to homogeneity as described previously $(18,22)$. The recombinant human Drosophila homologue CK2 $\alpha$-Timekeeper $\left(\mathrm{CK} 2 \alpha^{\mathrm{Tik}}\right)$ was expressed and purified essentially as described in ref. 23 .

Protein kinase assays. CK2 activity assay was performed in a reaction mixture containing the CK2-specific synthetic peptide RRRDDDSDDD essentially as described previously $(18,19)$.

AKT kinase assay was performed in vitro with human recombinant AKT1 using as a substrate either $1 \mu \mathrm{g}$ histone 2B (H2B, Roche) or a $100 \mu \mathrm{M}$ synthetic peptide (RPRAATF, Upstate Biotechnology) in an assay buffer containing $25 \mathrm{mM}$ Tris/ $\mathrm{HCl} \mathrm{pH}$ 7.5, $30 \mathrm{mM}$ ß-glycerophosphate, $10 \mathrm{mM} \mathrm{MgCl}$, $1 \mathrm{mM} \mathrm{NaVO}_{4}, 20 \mathrm{mM} \mathrm{NaF}, 1 \mathrm{mM}$ DTT, $50 \mu \mathrm{M}$ ATP and $5 \mu \mathrm{Ci}\left[\gamma-{ }^{32} \mathrm{P}\right] \mathrm{ATP}$. Reaction mixtures were incubated at $30^{\circ} \mathrm{C}$ for $30 \mathrm{~min}$. Assays performed in the presence of histone $2 \mathrm{~B}$ substrate were stopped by adding SDS-sample buffer. Samples were, thereafter, analyzed by SDS-PAGE. Radioactive bands were revealed by autoradiography. Assays performed in the presence of a synthetic peptide were stopped on ice and, immediately afterwards, spotted onto P81 phosphocellulose paper filters (Whatmann). Filters were washed three times with $0.75 \%(\mathrm{v} / \mathrm{v})$ phosphoric acid and subsequently transferred into vials containing a scintillation cocktail. Radioactivity was quantified by scintillation counting (Hewlett-Packard). When AKT kinase assays were performed in the presence of CK $2 \alpha$, CK $2 \alpha^{\text {Tik }}$, CK $2 \beta$ or CK2 holoenzyme, proteins were preincubated $30 \mathrm{~min}$ on ice with AKT prior performing the kinase assay. Alternatively, kinase assays with native AKT were performed after immunoprecipitation of AKT under the same 
conditions described above. Where indicated, H-89 dihydrochloride (Calbiochem) was used at $100 \mu \mathrm{M}$ concentration, PKA inhibitor peptide (PKI-[6-22]- $\mathrm{NH}_{2}$, Upstate) and emodin (Calbiochem) were used at $1 \mu \mathrm{M}$ and $20 \mu \mathrm{M}$ concentration, respectively.

Flow cytometry analysis. Apoptosis was quantified by flow cytometry by determining the percentage of cells in subG1. Cells were trypsinized, combined with floating cells present in the medium and fixed in $70 \%$ ethanol overnight at $-20^{\circ} \mathrm{C}$ after extensive washing with phosphate-buffered saline (PBS). Fixed cells were then incubated for $30 \mathrm{~min}$ with $20 \mu \mathrm{g} / \mathrm{ml}$ propidium iodide (Sigma) and $40 \mu \mathrm{g} / \mathrm{ml}$ RNase (Roche) in PBS prior to analysis. Cells were analyzed by a FACSCalibur flow cytometer (Becton-Dickinson Biosciences). Data were analyzed by Cell Quest Pro Analysis software.

\section{Results}

Inhibition of the PI3K pathway potentiates the nocodazoleinduced apoptotic response of cells treated with CK2 antisense oligodeoxynucleotides (ASO). In order to test the possibility that CK2 might act in synergism with AKT in protecting cells from apoptosis, HeLa cells were depleted of CK2 subunits by treatment with ASO directed against the catalytic $\alpha / \alpha^{\prime}$ and regulatory $\beta$ subunits prior induction of apoptosis. As shown in Fig. 1, cell treatment with ASO results in a drop of CK2 catalytic activity of about 50\% (Fig. 1A) (19) and an intracellular depletion of CK2ß subunit (Fig. 1B). The incubation of cells with CK2B-ASO does not affect the expression of CK2 catalytic subunits (Fig. 1B) under the condition applied. As shown in Fig. 1C, the treatment of HeLa cells with nocodazole leads to enhanced apoptosis compared to control cells (Fig. 1C, bars 1 and 2). The depletion of cells from CK2 catalytic subunits with CK $2 \alpha / \alpha^{\prime}-\mathrm{ASO}$ leads to a consistent increase in the nocodazole-induced apoptotic response as reported previously (19) and as shown in Fig. 1C, bars 5 and 6 . Additionally in this study, it is apparent that the treatment of cells with CK2ß-ASO (Fig. 1B) leads to a similar effect (Fig. 1C, bars 8 and 9) although the treatment of cells with CK $2 \alpha / \alpha^{\prime}$-ASO seems to be more effective than the one with CK2ß-ASO in enhancing the apoptotic response induced by nocodazole. The treatment of cells with ASO against CK2 evokes a consistent apoptotic response in a variety of different cell lines and suggests that their effect might be further enhanced by the combination with other factors e.g. inhibitors which block the activity of protein kinases involved in the apoptosis signalling. Recent studies have shown that wortmannin, a fungal metabolite, has a potent anti-tumor activity in vitro as well as in vivo promoting apoptosis by inhibiting PI3K with high selectivity (24). As shown in Fig. 1, the induction of apoptosis by nocodazole in cells pre-treated for $1 \mathrm{~h}$ with wortmannin leads to a $58.5 \%$ increment in the apoptotic response compared to cells treated with nocodazole only (Fig. 1C, bars 2 and 4) and to a $68.7 \%$ increment in the percentage of apoptotic cells treated with wortmannin only (Fig. 1C, bar 3). Moreover, when cells are depleted of CK2 subunits by ASO and pre-incubated with wortmannin prior to the induction of apoptosis, a further consistent increase in the apoptotic response is observed. Cells depleted of CK2
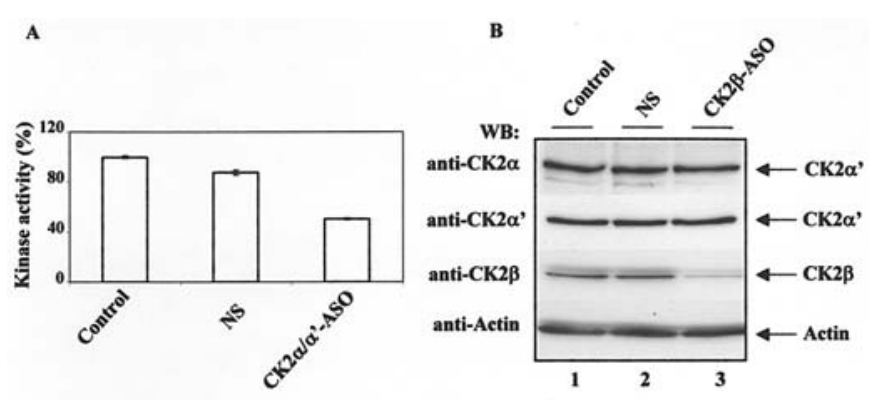

C

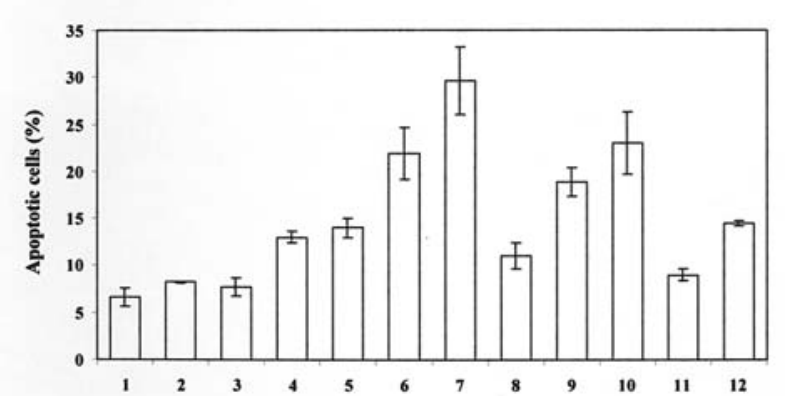

Figure 1. Wortmannin enhances nocodazole-induced apoptosis in HeLa cells treated with CK2 ASO. (A), Protein kinase CK2 activity test on total lysates from untreated HeLa cells (control), HeLa cells treated with nonsense oligodeoxynucleotide (NS) or with $\mathrm{CK} 2 \alpha / \alpha^{\prime}$ ASO (CK $\left.2 \alpha / \alpha^{\prime}-\mathrm{ASO}\right)$, respectively. Results are presented as percentage of kinase activity relative to that of untreated (control) cells. (B), Total lysate from HeLa cells untreated (lane 1) or treated with nonsense oligodeoxynucleotide (NS) (lane 2) or CK2ß-ASO (lane 3), respectively, were subjected to Western blot analysis using the indicated antibodies. (C), HeLa cells were left untreated (bar 1); treated with $400 \mathrm{ng} / \mathrm{ml}$ nocodazole for $24 \mathrm{~h}$ (bar 2); $100 \mathrm{nM}$ wortmannin (bar 3); nocodazole and wortmannin (bar 4); CK2 $\alpha / \alpha^{\prime}-\mathrm{ASO}$ (bar 5); nocodazole and CK $2 \alpha / \alpha^{\prime}-\mathrm{ASO}$ (bar 6); nocodazole, wortmannin and CK2 $\alpha / \alpha^{\prime}$-ASO (bar 7); CK2ß-ASO (bar 8); nocodazole and CK2ß-ASO (bar 9); nocodazole, wortmannin and CK2ß-ASO (bar 10); NS (bar 11) and nocodazole, wortmannin and NS (bar 12), respectively. Cell treatments were performed as described under Materials and methods and apoptosis was quantified by flow cytometry. Statistical analysis was performed on data collected from three independent experiments.

catalytic subunits and pre-treated with wortmannin show a $35 \%$ increase in nocodazole-induced apoptosis compared to the corresponding control experiment (Fig. 1C, bars 6 and 7) while in the case of cells depleted of CK2ß, the pre-treatment with wortmannin leads to a $22 \%$ increase in apoptosis (Fig. 1C, bars 9 and 10). Fig. 1C also shows a control experiment where the induction of apoptosis which follows cell treatment with a nonsense oligodeoxynucleotide and wortmannin (Fig. 1C, bars 11 and 12) evokes a response comparable with the one shown in Fig. 1C, bar 4. The analysis of cells, depleted of $\mathrm{CK} 2 \alpha / \alpha^{\prime}$ or $\beta$ subunits and treated with wortmannin, did not lead to a significant change in the percentage of apoptotic cells compared to cells treated with ASO only (results not shown).

AKT interacts with CK2 subunits in vivo as well as in vitro. Compounds which negatively regulate PI3K act through the so-called PI3K-AKT pathway. As in the case of CK2, AKT has been implicated in the modulation of cell proliferation and cell survival through phosphorylation of numerous substrate targets. Therefore, the ability of CK2 subunits to form stable complexes with AKT in vivo was assessed. The transient expression of HA-AKT and either of the CK2 subunits was 


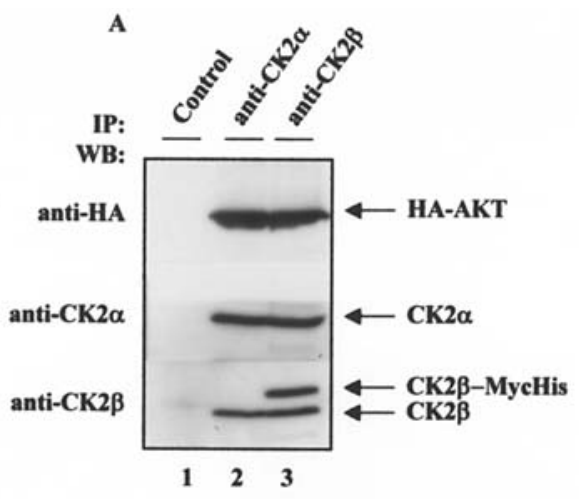

B
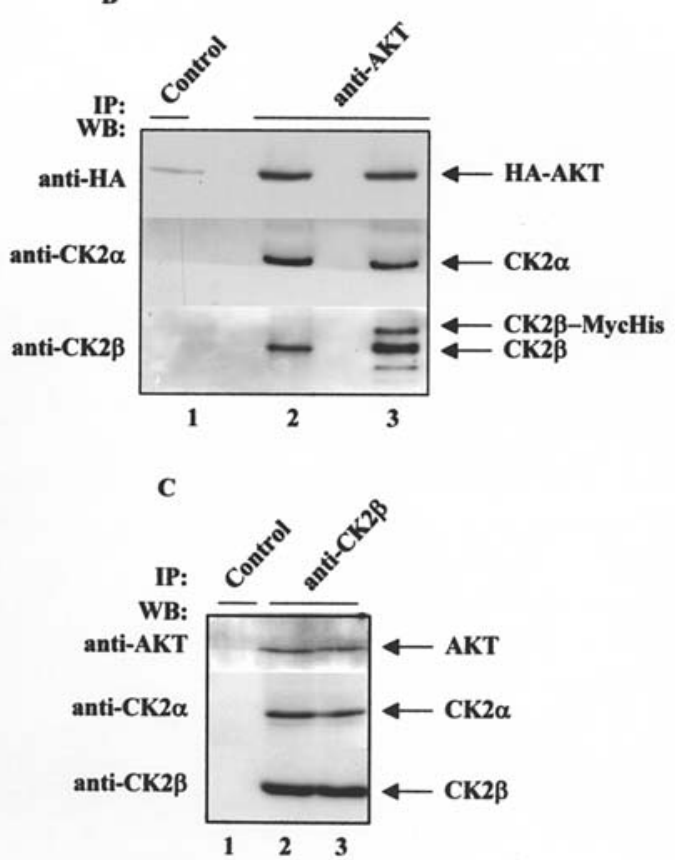

Figure 2. The AKT kinase interacts with CK2 subunits in vivo. (A), Cos-1 cells were transiently transfected with either HA-AKT and CK2ß-MycHis (lanes 1 and 3) or HA-AKT and CK2 $\alpha$ plasmids (lane 2). Total lysates were subjected to immunoprecipitation (IP) using the indicated rabbit polyclonal antibodies. Lane 1 shows a control experiment where cells transiently co-expressing HA-AKT and CK2B-MycHis were subjected to immunoprecipitation with control serum. Immunoprecipitates were then analyzed by Western blot using the indicated mouse monoclonal antibodies. (B), The experiment was performed essentially as described in (A). Cos-1 cells were transfected with either HA-AKT and CK2 $\alpha$ (lane 2) or HA-AKT and CK2ß-MycHis (lanes 1 and 3) plasmids. The immunoprecipitation was performed with rabbit polyclonal anti-AKT antibody. (C), Total cell extracts from K562 (lane 2) and HL-60 (lane 3 ) cells were subjected to immunoprecipitation using a rabbit polyclonal anti-CK $2 \beta$ serum. Lane 1 shows a control experiment performed with a lysate from K562 cells in the presence of control serum. Proteins were analyzed by Western blot using the indicated antibodies.

induced by transfecting Cos- 1 cells with the corresponding plasmid DNAs. To determine whether AKT associates with CK2, immnunoprecipitation experiments were performed using polyclonal anti-CK2 $\alpha$ (Fig. 2A, lane 2) and polyclonal anti-CK2ß (Fig. 2A, lane 3) antibodies, respectively. Proteins were separated by SDS-PAGE and analyzed by Western blot and the membranes were then probed with the indicated antibodies. As shown in Fig. 2A, both polyclonal antibodies (i.e. anti-CK2 $\alpha$ and anti-CK2 3 ) are able to co-precipitate AKT kinase. As expected, the immunoprecipitation performed with

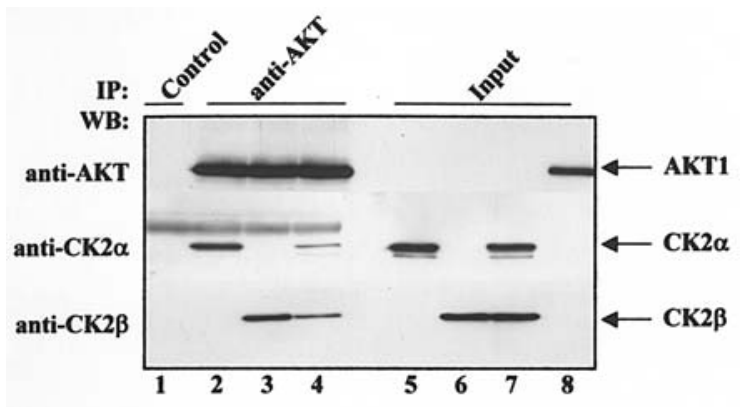

Figure 3. Recombinant AKT kinase forms a complex with CK2 subunits in vitro. Recombinant AKT1 (4 pmoles) was mixed with an equimolar amount of recombinant $\mathrm{CK} 2 \alpha$ (lane 2), recombinant CK2ß (lane 3), or recombinant CK2 holoenzyme (lane 4), respectively. The complexes were immunoprecipitated (IP) with a rabbit polyclonal anti-AKT antibody. Lane 1 represents a control experiment performed with a rabbit polyclonal control serum in the presence of equimolar amounts of AKT and CK2 holoenzyme. Lanes 5-8 show the detection of 2 pmoles of purified recombinant CK $2 \alpha$, CK2 $2 \beta$, CK 2 holoenzyme and AKT1, respectively. Proteins were analyzed by Western blot using the indicated mouse monoclonal antibodies.

polyclonal anti-CK2 $\alpha$ (Fig. 2A, lane 2) and polyclonal antiCK2ß (Fig. 2A, lane 3) antibodies leads to the co-precipitation of either endogenous CK $2 \beta$ and endogenous CK $2 \alpha$. Next, formation of the AKT-CK2 complex was verified by immunoprecipitation with a polyclonal anti-AKT antibody (Fig. 2B). Cells transfected with either HA-AKT and CK2 $\alpha$ (Fig. 2B, lane 2) or HA-AKT and CK2ß-MycHis (Fig. 2B, lanes 1 and 3) plasmids, were subjected to immunoprecipitation and proteins were analyzed as described above. Results shown in Fig. 2B, indicate that AKT seems to interact with the CK2 holoenzyme although there might be a preferential interaction with $\mathrm{CK} 2 ß$. According to the intensity of the detected bands, while the amount of CK $2 \alpha$ interacting with AKT does not change whether CK $2 \alpha$ is overexpressed (lane 2) or endogenous (lane 3), the total amount of CK2ß in complex with AKT is higher in lane 3. Thus, if AKT interacted exclusively with CK2 holoenzyme one would expect to detect a higher amount of CK $2 \alpha$ in lane 3 than in lane 2.

To verify whether AKT-CK2 complex formation also occurs in cells not transiently overexpressing AKT or CK2, lysates from K562 and HL-60 cells were subjected to immunoprecipitation with polyclonal anti-CK2ß antibody (Fig. 2C). The immunoprecipitates were analyzed by Western blot performed after protein separation by SDS-PAGE and probed with the indicated antibodies (Fig. 2C). Results obtained using the indicated cell lines show that the complex formation between AKT and CK2 occurs under physiological conditions. Next, it was verified whether the interaction between AKT and CK2 subunits occurs also in vitro (Fig. 3). Equimolar amounts of either purified recombinant AKT and CK2 $\alpha$ (Fig. 3, lane 2), AKT and CK2B (Fig. 3, lane 3) or AKT and CK2 holoenzyme (Fig. 3, lanes 1 and 4), were subjected to immunoprecipitation with polyclonal anti-AKT antibody. Lanes 5-8 show the detection of 2 pmoles of pure CK2 $\alpha$ (lane 5), CK2 3 (lane 6), CK2 holoenzyme (lane 7) and AKT1 (lane 8), respectively. By comparison based on the results shown in lanes $1-4$, it can be concluded that 4 pmoles of AKT are immunoprecipitated in each experiment (lanes 2-4) and that about 1 pmol of CK2 $\alpha$ (lane 2) and 1 pmol CK2ß (lane 3) are co- 


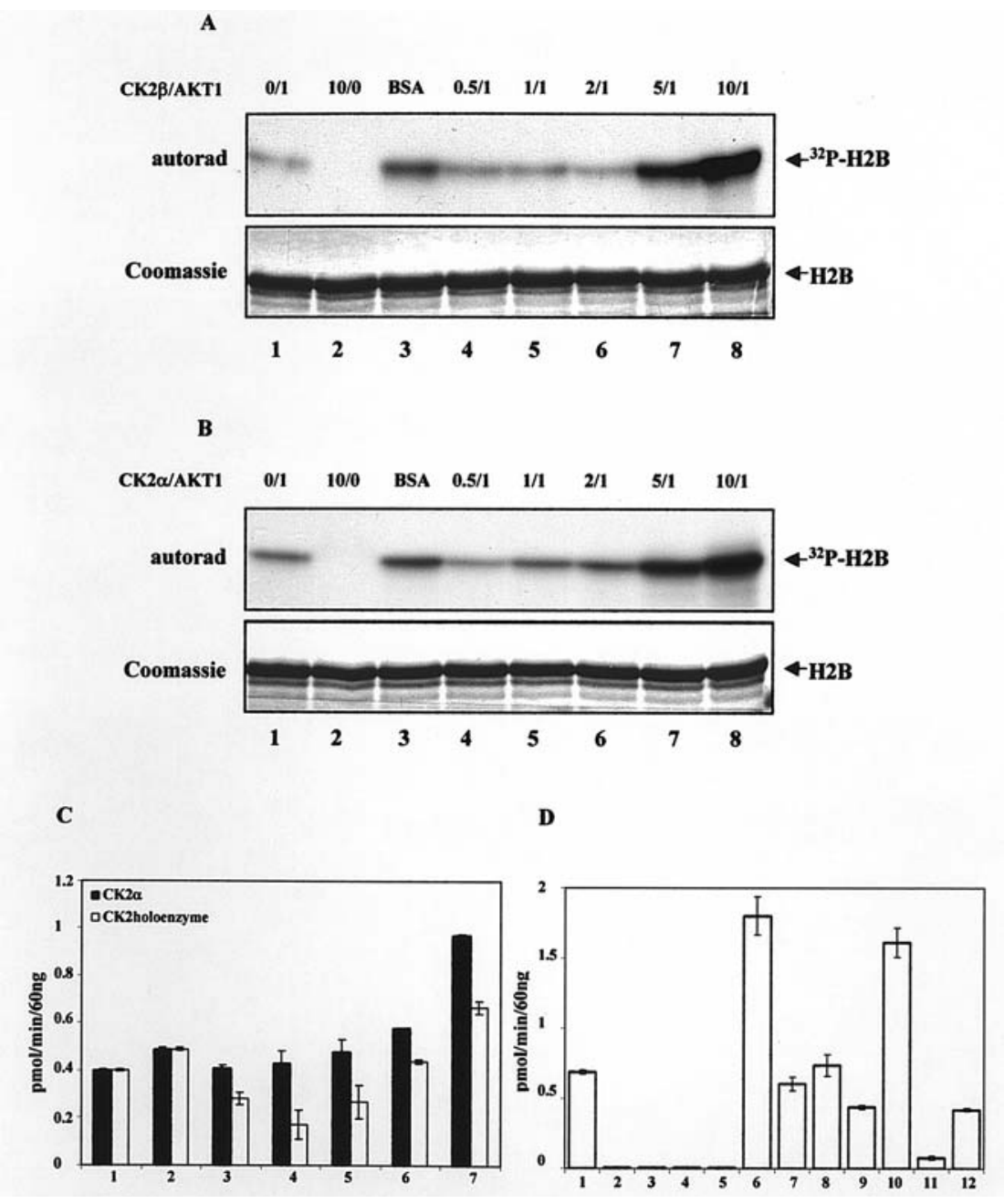

Figure 4. The interaction of AKT with CK2 subunits enhances AKT kinase activity in vitro. (A), AKT kinase assay was performed in the presence of 1 pmol recombinant AKT (lane 1) or 1 pmol recombinant AKT and increasing amounts of recombinant CK2ß (lane 4, 0.5 pmol; lane 5, 1 pmol; lane 6, 2 pmoles; lane 7, 5 pmoles and lane 8,10 pmoles, respectively). Lanes 2 and 3 are control experiments where either 10 pmoles CK2 3 alone (lane 2 ) or 1 pmol AKT together with 10 pmoles BSA (lane 3), were incubated with the reaction mixture. At the top of each panel the ratio CK2B/AKT (pmol) is indicated. (B), The experiment was performed essentially as described in (A) except that increasing amounts of recombinant CK2 $\alpha$ were incubated with 1 pmol AKT. (C), Bar graph showing the influence of increasing amount of either CK2 $\alpha$ (closed bars) or CK2 holoenzyme (open bars) on AKT kinase activity. In bar 1, the phosphorylation of AKT synthetic peptide in the presence of 1 pmol AKT is shown; in bar 2, the AKT kinase activity (1 pmol) is tested in the presence of 10 pmoles BSA; bars 3-7 show the influence that increasing amounts of either CK2 $\alpha$ or CK2 holoenzyme (bar 3, 0.5 pmol; bar 4, 1 pmol; bar 5, 2 pmoles; bar 6, 5 pmoles; bar 7, 10 pmoles) have on AKT (1 pmol) kinase activity. (D), Control AKT kinase assay. Bar 1, refers to the incubation of 1 pmol AKT with AKT peptide substrate; bar 2, refers to the incubation of AKT in the absence of AKT peptide substrate peptide; bar 3 , shows the incubation of 10 pmoles CK2 $\alpha$ with the reaction mix (absence of AKT and AKT substrate peptide); bar 4, shows the incubation of 10 pmoles CK2 $\alpha$ with AKT peptide substrate only; bars 5 and 6 , refer to the incubation of AKT with CK2 $\alpha$ in the absence and in the presence, respectively, of AKT peptide substrate; bars 7-10, refer to experiments performed as described for bars 3-6 but in the presence of CK2 holoenzyme instead of CK2 $\alpha$. Bars 11 and 12 refer to the incubation of AKT with AKT substrate peptide, $100 \mu \mathrm{M}$ AKT inhibitor H-89 and either CK2 $\alpha$ (bar 11) or CK2 holoenzyme. The values indicate the total pmoles of phosphate incorporated into the AKT peptide substrate/ $\mathrm{min} / \mathrm{pmol} \mathrm{AKT}(60 \mathrm{ng})$ and represent the mean $\pm \mathrm{SD}$ of three independent experiments.

immunoprecipitated with AKT1 while less than 0.5 pmol of CK2 holoenzyme (lane 4) interact with AKT1. Results shown in Fig. 3 support the in vivo findings i.e. AKT interacts with the individual $\mathrm{CK} 2$ subunits (i.e. CK2 $\alpha$ and $C K 2 \beta$ ) and the AKT-CK2 holoenzyme interaction is possibly weaker.

The interaction of CK2 subunits with AKT leads to enhanced AKT kinase activity. In order to assess whether the interaction of CK2 with AKT might have an effect on AKT kinase activity, an in vitro kinase assay was performed where 1 pmol AKT was incubated with increasing amounts of purified recombinant $\mathrm{CK} 2 \beta$ (i.e. from 0.5 pmol up to 10 pmoles, Fig. 4A, lanes 4-8) and tested for its ability to phosphorylate histone $2 \mathrm{~B}$, a protein routinely used for determining AKT kinase activity. Proteins were resolved by SDS-PAGE and the phosphate incorporation on histone $2 \mathrm{~B}$ revealed by autoradiography. As shown in Fig. 4A, AKT-catalyzed histone 2B phosphorylation is enhanced (the signal intensity of the band shown in lane 8 is estimated to be about 4 -fold higher than the one shown in lane 3 ) in the presence of increasing amount of CK2ß. Although a slight AKT-activating effect is also observed in the presence of 10 pmoles BSA (Fig. 4A, lane 3), the experiment nevertheless clearly shows that the presence of $C K 2 B$ upregulates AKT kinase with respect to histone $2 \mathrm{~B}$ phosphorylation. Results shown in Fig. 4B indicate that the presence of recombinant CK2 $\alpha$ exerts a similar effect inasmuch as the kinase activity of AKT increases (the signal intensity of the band shown in lane 8 is estimated to be about 2.5 -fold 
higher than the one shown in lane 3) with the concomitant increase of CK $2 \alpha$. A kinase assay performed in the presence of AKT and CK2 holoenzyme revealed that the CK2 holoenzyme but not CK2 $\alpha$ was able to phosphorylate a contaminant protein present in the histone 2B preparation (it is well-known that CK2ß mediates not only the interaction between the catalytic subunits but also the selectivity towards some CK2 substrate targets) with a molecular mass very similar to the histone $2 \mathrm{~B}$ making it difficult to evaluate AKT-catalyzed histone $2 \mathrm{~B}$ phosphorylation (results not shown). An AKTkinase assay using a synthetic peptide as AKT substrate (Fig. 4C) was therefore used. The experiment was performed in the presence of AKT and either increasing amounts of CK2 holoenzyme (Fig. 4C, open bars) or, for comparison, CK2 $\alpha$ (Fig. 4C, solid bars), respectively. The kinase assay confirmed that CK2 $\alpha$ enhances the AKT kinase activity while the presence of CK2 holoenzyme does not lead to an effect of the same extent.

A further indication that the presence of the individual CK2 subunits and not the CK2 holoenzyme plays a major role in the regulation of AKT kinase is shown in Fig. 4D. A control experiment was performed where AKT kinase activity was measured in the presence of either CK2 $\alpha$ (bars 5 and 6) and CK2 holoenzyme (bars 9 and 10) and in the presence (bars 6 and 10) or absence (bars 5 and 9) of AKT synthetic peptide, respectively. Results indicate that while the increased AKT kinase activity in the presence of $\mathrm{CK} 2 \alpha$ is a specific AKT-upregulation (compared to the control experiment indicated in bar 1) measured by the specific AKT-catalyzed phosphorylation of the synthetic peptide (Fig. 4D, bar 6), the same conclusion cannot be drawn in the case of the CK2 holoenzyme (Fig. 4D, bars 9 and 10) indicating that the CK2 holoenzyme does not influence to the same extent as the individual CK2 subunits the activity of AKT. Fig. 4D shows also control experiments: bar 2 refers to AKT incubated in a reaction mix in the absence of AKT peptide substrate while bars 3 and 7 refer to CK $2 \alpha$ and CK2 holoenzyme, respectively, incubated without AKT and AKT peptide substrate. Bars 4 and 8 refer to CK $2 \alpha$ and CK2 holoenzyme, respectively, incubated without AKT and in the presence of AKT peptide substrate. Bars 11 and 12 refer to AKT incubated with CK2 $\alpha$ and CK2 holoenzyme, respectively, in the presence of AKT substrate peptide and H-89 a known inhibitor of AKT but not of CK2 (25). The analysis of the collected data confirm the observation that the enhanced AKT kinase activity seen in the presence of CK2 holoenzyme is only partially due to the specific phosphorylation of the synthetic peptide by AKT.

Given the fact that the presence of CK2 subunits enhances to similar extent the kinase activity of AKT, it was assessed whether or not the observed effect was dependent on CK $2 \alpha$ catalytic activity. Human recombinant CK $2 \alpha$ was mixed with human recombinant AKT1 and the degree of AKT activation was compared to the one in the presence of a variant of CK $2 \alpha$ called Timekeeper (CK2 $\left.\alpha^{\mathrm{Tik}}\right)$. CK $2 \alpha^{\mathrm{Tik}}$ kinase activity is severely impaired by the presence of two point mutations i.e. Met161Lys and Glu165Asp (Fig. 5A) $(23,26)$. As shown in Fig. 5B, the AKT1-catalyzed phosphorylation of histone 2B in the presence of either CK $2 \alpha$ or $\mathrm{CK} 2 \alpha^{\mathrm{Tik}}$ is comparable indicating that the interaction of $\mathrm{CK} 2 \alpha$ with AKT plays a role in the modulation of AKT kinase activity in vitro.
$\mathbf{A}$

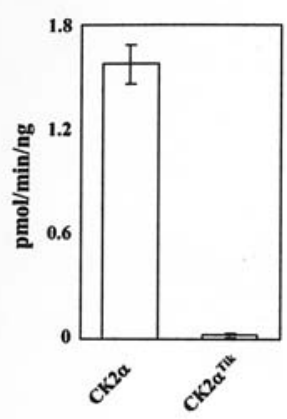

B

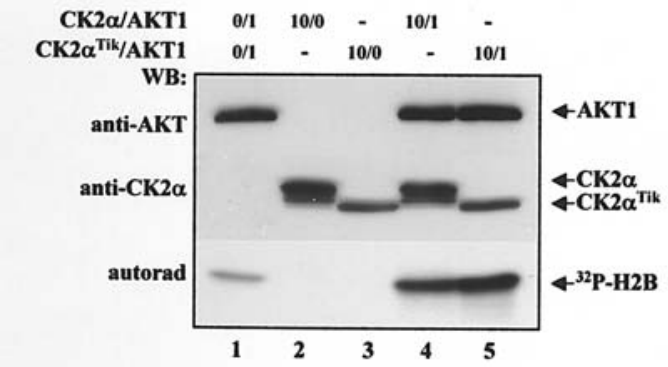

Figure 5. The upregulation of AKT activity is independent of CK $2 \alpha$ kinase activity. (A), Enzyme activity comparison between human recombinant CK $2 \alpha$ wild-type and human recombinant Timekeeper mutant CK2 $\alpha^{\text {Tik }}$. (B), AKT kinase assay performed in the presence of 1 pmol human recombinant AKT1 (lane 1) or 1 pmol human recombinant AKT1 and 10 pmoles of either recombinant CK2 $\alpha$ wild-type (lane 4) or CK $2 \alpha^{\text {Tik }}$ (lane 5). Lanes 2 and 3 are control experiments where $\mathrm{CK} 2 \alpha$ (lane 2 ) and $\mathrm{CK} 2 \alpha^{\text {Tik }}$ (lane 3 ) were incubated in the absence of AKT kinase with the reaction mixture, respectively.

The modulation of AKT activity by CK2 subunits was also determined in vivo using two different cell models. Cells overexpressing CK2ß-Myc in a tetracycline-regulated manner were transfected with HA-AKT. After $24 \mathrm{~h}$ from initial transfection, the cells were incubated for $24 \mathrm{~h}$ in serum-free medium. AKT activation was induced, where indicated, by adding IGF-1 as described under Materials and methods. Lysates from cells expressing active AKT only (Fig. 6A, lane 1) or active AKT and CK2ß-Myc (Fig. 6A, lane 2) were subjected to immunoprecipitation experiments in the presence of polyclonal anti-AKT (Fig. 6A, lane 1) and polyclonal antiCK2ß (Fig. 6A, lane 2) antibodies, respectively. As shown in Fig. 6A, the phosphorylation of histone $2 \mathrm{~B}$ by HA-AKT in the anti-AKT immune complex (lane 1) is lower than the one observed by HA-AKT in the anti-CK2ß complex (lane 2). Considering the amounts of HA-AKT precipitated in the two experiments shown in Fig. 6A, it is apparent that the complex formation between AKT and CK2B leads to enhanced AKT kinase activity. The activation of AKT kinase in the AKT/ CK2B complex was confirmed by immunoprecipitation experiments performed in the presence of polyclonal antiAKT antibody (Fig. 6B). As for the previous experiment, coexpression of HA-AKT and CK2B-Myc leads to increased histone $2 \mathrm{~B}$ phosphorylation catalyzed by $\mathrm{AKT}$ in complex with CK2ß (lane 3). To rule out the possibility that the observed 

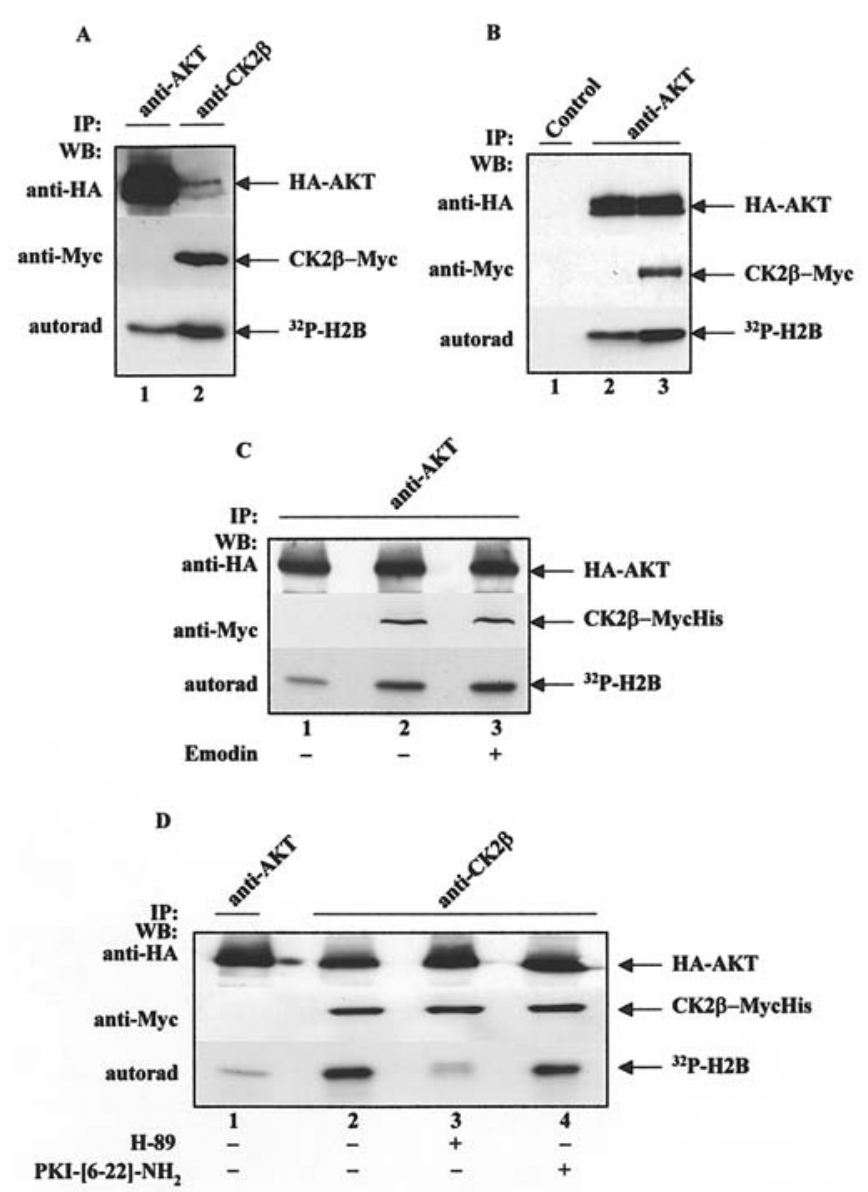

Figure 6. AKT in complex with $\mathrm{CK} 2 \beta$ has a higher kinase activity in vivo. (A), GV10.15 cells stably expressing Myc-tag CK2ß were transfected with HA-AKT plasmid. Lane 1 refers to cells transiently expressing AKT while lane 2 refers to cells co-expressing AKT and CK2ß-Myc. Cell extracts were subjected to immunoprecipitation as indicated in the upper part of the panel. The precipitates were assayed for AKT kinase activity using histone $2 \mathrm{~B}$ as substrate. Samples were resolved by SDS-PAGE and subsequently analyzed by Western blot with the indicated mouse monoclonal antibodies. ${ }^{32} \mathrm{P}$ incorporation was analyzed by autoradiography. (B), The experiment was performed as in (A) except that the immunoprecipitation was performed with rabbit polyclonal anti-AKT antibody (lanes 2 and 3). Lane 1 shows a control experiment where a lysate from cells co-expressing HA-AKT and CK2ß-Myc was subjected to immunoprecipitation with a control serum. (C), Cos-1 cells were transfected with either HA-AKT (lane 1), or HA-AKT and CK2ß-MycHis plasmids (lanes 2 and 3). Protein extracts were subjected to immunoprecipitation with the indicated antibody. AKT kinase activity was tested in the presence of histone $2 \mathrm{~B}$ as substrate and in the absence (lanes 1 and 2) or in the presence (lane 3) of $20 \mu \mathrm{M}$ emodin. (D), Cos-1 cells were transfected with either HAAKT (lane 1) or HA-AKT and CK2ß-MycHis plasmids (lanes 2-4), respectively. Lysates were subjected to immunoprecipitation with the indicated antibodies. AKT kinase activity was tested with histone $2 \mathrm{~B}$ as substrate in the absence (lanes 1 and 2) or in the presence of $100 \mu \mathrm{M} \mathrm{H}-89$ (lane 3) and $1 \mu \mathrm{M}$ PKI-[6-22]- $\mathrm{NH}_{2}$ (lane 4), respectively. In all experiments the activation of AKT was induced as described under Materials and methods. Data are representative of three independent experiments.

increased phosphorylation of histone $2 \mathrm{~B}$ was not due to the presence of CK2 $\alpha$ or cAMP-dependent protein kinase (PKA) non-specifically bound to the complex AKT-CK2ß, Cos-1 cells were subjected to immunoprecipitation essentially as indicated in Fig. 6A and B. It followed an AKT-kinase assay in the absence (Fig. 6C, lanes 1 and 2; Fig. 6D, lanes 1 and 2) and in the presence of emodin an effective inhibitor of CK2 (Fig. 6C, lane 3) (27), H-89 inhibitor (Fig. 6D, lane 3) or PKA inhibitor peptide (Fig. 6D, lane 4), respectively. The

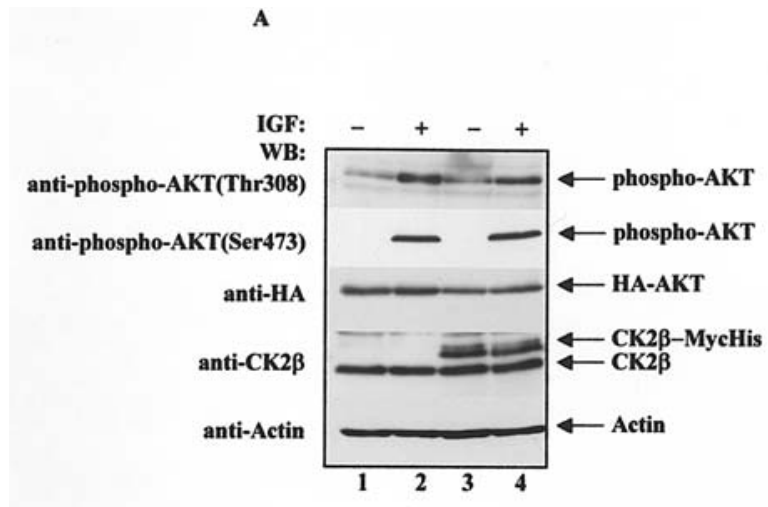

B

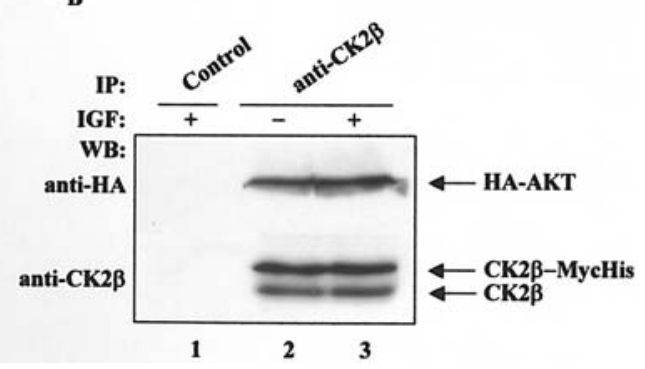

Figure 7. The overexpression of CK2 3 does not enhance the activity of PDK1 and the phosphorylation status of AKT does not influence the AKT-CK2B complex formation. (A), Cos-1 cells overexpressing HA-AKT (lanes 1 and 2) or HA-AKT and CK2ß-MycHis (lanes 3 and 4) were stimulated with $50 \mathrm{ng} / \mathrm{m}$ IGF-1 for $10 \mathrm{~min}$ as indicated in the figure. Cells lysates were separated by SDS-PAGE and proteins were analyzed by Western blot using the indicated antibodies. The phosphorylation of AKT was detected using rabbit polyclonal anti-phospho-AKT(Thr308) and mouse monoclonal anti-phospho-AKT(Ser473) antibodies as indicated. (B), Whole cell extracts from cells transfected as described in (A) were subjected to immunoprecipitation using a rabbit polyclonal anti-CK2B antibody (lanes 2 and 3). Proteins were then analyzed as described above. A control experiment where cells transiently co-expressing active HA-AKT and CK2ß-MycHis were subjected to immunoprecipitation with control serum, is shown in lane 1.

presence of $\mathrm{H}-89$ abolishes the AKT-catalyzed histone 2B phosphorylation while the presence of PKI-[6-22]- $\mathrm{NH}_{2}$ [known to be the shortest synthetic PKI analog that inhibits PKA with the highest potency $\left(\mathrm{K}_{\mathrm{i}}\right.$ value of $\left.1.7 \mathrm{nM}\right)$ (28)] and emodin does not change significantly the phosphorylation level of the AKT substrate target. The data reinforce the notion that the phosphorylation of histone $2 \mathrm{~B}$ by AKT in complex with CK2ß might be specific.

AKT/CK2 3 complex formation is not enhanced upon IGF-1mediated AKT activation. Although experiments performed in vitro indicate the ability of CK2 subunits to directly enhance the activity of AKT (Fig. 4), one should not exclude the possibility that the induction of CK $2 \beta$ overexpression in vivo could lead to stimulation of AKT-upstream mediators of the PI3K signaling pathway contributing to enhance AKT activity. The activation of AKT takes place at the plasma membrane where AKT is phosphorylated and thus stabilized at two residues: Thr308 and Ser473, the former targeted by PDK1 the latter by mTOR kinase (29). In order to assess whether the expression of CK2ß enhances the activity of AKT-upstream kinases, the phosphorylation of AKT was verified by Western blot analysis using phospho-specific AKT antibodies as indicated in Fig. 7. Results shown in Fig. 7A indicate that 
the overexpression of CK2B does not lead to enhanced PDK1/ mTOR kinase activity (Fig. 7A, lanes 2 and 4).

As reported above, the fact that the activation of AKT (which follows cell stimulation with IGF-1) leads to AKT phosphorylation and stabilization of its active conformation, suggested to examine whether the phosphorylation status of AKT might influence its association with CK2ß. Immunoprecipitation experiments with cell lysates expressing CK2ß and AKT in the inactive (Fig. 7B, lane 2) and in the active (Fig. 7B, lanes 1 and 3) state, respectively, suggest that IGF-1mediated AKT phosphorylation does not modulate the interaction between CK2ß and AKT in vivo.

\section{Discussion}

Although CK2 is traditionally considered to be an enzyme with a tetrameric structure, there is plenty of evidence indicating that in cells CK2 is present in a dynamic equilibrium with the individual subunits (reviewed in ref. 30). The crystal structure determination of human recombinant CK2 (31) has indeed reinforced this concept revealing that the CK $2 \alpha / \beta$ interface is relatively small if compared with the one typically observed in stable protein complexes. The literature has numerous examples where the individual CK2 subunits form stable complexes with other proteins and protein kinases (reviewed in ref. 30). In this respect, it is interesting to note that the interaction of CK2 with other protein kinases does not lead always to the same effect (in terms of activation/ inhibition of the interacting protein kinase). While, for instance, the interaction of CK2ß with c-Mos inhibits c-Mos kinase activity (32), the complex between CK2ß and the checkpoint kinase Chk1 upregulates Chk1 kinase activity (18).

In this study, it is shown for the first time that CK2 interacts with AKT in vitro as well as in vivo. The complex formation with CK2 individual subunits seems to be more specific then the one with the CK2 tetrameric form. This might explain the fact that the association between AKT and either CK $2 \alpha$ or CK2ß markedly enhances AKT kinase activity in vitro as well as in vivo (the latter demonstrated in the case of CK $2 \beta$ overexpression in cells) while an effect to the same extent is not observed in the case of CK2 holoenzyme. In the latter case, in vitro experiments show that CK2 holoenzyme seems to have an effect (in terms of measured radioactivity) that is independent of the presence of AKT and AKT peptide substrate indicating that the observed activation of AKT by CK2 might be due, at least in part, to an unspecific detection of CK2 autophosphorylation (Fig. 4D).

In order to demonstrate that the regulatory interaction between AKT and the individual CK2 subunits may have physiological relevance, two different cell lines were employed where AKT activity was measured after induction of CK2 3 overexpression. Although the employment of protein kinase inhibitors selectively targeting AKT, CK2 and PKA (often found associated with AKT precipitates), respectively, confirms the data obtained in vitro, it cannot be completely excluded that a contaminant protein kinase, present in the immunoprecipitates, might contribute to the upregulation of AKT.

While this manuscript was in preparation, Di Maira et al (33) reported that protein kinase CK2 phosphorylates and upregulates AKT kinase. Although the data presented in this paper indicate that $\mathrm{CK} 2 \alpha$-dependent upregulation of AKT is independent from CK $2 \alpha$ kinase activity, collectively these observations indicate that CK2 might play a pivotal role in the regulation of AKT activity through two distinct mechanisms: protein phosphorylation and protein-protein interaction. In this respect, a number of AKT-binding proteins have been identified in the last few years (reviewed in ref. 34). Their ability to up- or down-regulate AKT kinase suggests that the modulation of AKT activity is not exclusively dependent on site-specific phosphorylation.

Various lines of evidence indicate that the deregulated CK2 expression in mammalian cells and in animal models increases the oncogenic potential of cells especially in cooperation with other factors such as c-Myc, Tal-1 and Ha-Ras (reviewed in ref. 30). In line with these observations, several studies have shown that the treatment of tumor cells with ASO directed against CK2 catalytic subunits causes a strong reduction in cell proliferation which correlates with an enhanced apoptotic response. Recently, through the use of a xenograft model of prostate cancer, it has been shown that a single dose of CK2 $\alpha$-ASO given directly to the xenograft tumor in the nude mouse was sufficient to resolve the tumor because of potent cell induction of apoptosis (35).

Similarly, in the last 6-7 years accumulating evidence has clearly shown that uncontrolled activation of AKT either by gene amplification of AKT and/or AKT-upstream protein kinases or loss of PTEN, promotes tumor progression. AKT has been shown to be implicated in cell survival by delaying cell death and/or conferring resistance to cells treated with chemotherapeutic drugs, irradiation, ischemic shock etc. AKT positively regulates cell survival in different ways such as through the phosphorylation of intracellular proteins that are directly or indirectly implicated in the regulation of the apoptotic machinery, by indirectly regulating gene transcription, or by enhancing the degradation of pro-apoptotic proteins. An attractive idea is that AKT may play a prominent role in tumor progression or, more generally, in cell proliferation, acting in concert with proteins involved in survival pathways distinct from the PI3K-AKT one.

In this study, it is reported for the first time that the suppression of the PI3K-AKT pathway by cell treatment with wortmannin in combination with the depletion of cells of either CK2 subunits by ASO evokes a strong synergistic apoptotic response. The fact that the depletion of the individual CK2 subunits evokes an apoptotic response in cancer cells is in agreement with earlier studies (36) and, beside demonstrating the effect of deregulated expression of CK2, suggests that CK2 catalytic and regulatory subunits may negatively influence the apoptotic machinery in different ways; the former through protein-protein interaction and/or phosphorylation and the latter by protein association/dissociation.

The functional interaction between AKT and CK2 subunits provides additional insights into how the apoptotic machinery is regulated and underlines the importance of developing efficient strategies (e.g. design of inhibitors with high selectivity and novel approaches for an efficient delivery of small interfering molecules) to directly inhibit AKT and CK2 as a valuable therapeutic approach in cancer therapy. 


\section{Acknowledgments}

This work was supported by the Danish Cancer Society (grant no. DP 03093 to B.G.) and the Danish Research Council (grant no. 21-03-0508 to B.G.). The author is particularly thankful to Dr O.-G. Issinger for providing purified human recombinant AKT1, pBEHCK $2 \alpha$ and pT7-7CK $2 \alpha^{\text {Tik }}$ constructs and for comments on the manuscript and to Dr J.Y.J. Wang for the generous gift of HA-tag AKT plasmid and in whose laboratory the preliminary data were obtained. A special thanks to Dr D. Litchfield for providing GV10.15 cell line, to Dr G. Schoenhals for critical reading of the manuscript and to T. Holm for technical assistance.

\section{References}

1. Tawfic S, Yu S, Wang S, Faust R, Davis A and Ahmed K: Protein kinase CK2 signal in neoplasia. Histol Histopathol 16: 573-582, 2001.

2. Faust M and Montenarh M: Subcellular localization of protein kinase CK2. A key to its function? Cell Tissue Res 301: 329-340, 2000.

3. Seldin DC and Leder P: Casein kinase II alpha transgene-induced murine lymphoma: relation to theileriosis in cattle. Science 267 : 894-897, 1995.

4. Landesman-Bollag E, Channavajhala PL, Cardiff RD and Seldin DC: p53 deficiency and misexpression of protein kinase CK2alpha collaborate in the development of thymic lymphomas in mice. Oncogene 16: 2965-2974, 1998.

5. Kelliher MA, Seldin DC and Leder P: Tal-1 induces T cell acute lymphoblastic leukemia accelerated by casein kinase II alpha. EMBO J 15: 5160-5166, 1996.

6. Channavajhala PL and Seldin DC: Functional interaction of protein kinase CK2 and c-Myc in lymphomagenesis. Oncogene 21: 5280-5288, 2002.

7. Buchou T, Vernet M, Blond O, Jensen HH, Pointu H, Olsen BB, Cochet $\mathrm{C}$, Issinger $\mathrm{O}-\mathrm{G}$ and Boldyreff $\mathrm{B}$ : Disruption of the regulatory beta subunit of protein kinase CK2 in mice leads to a cell-autonomous defect and early embryonic lethality. Mol Cell Biol 23: 908-915, 2003.

8. Chang F, Lee JT, Navolanic PM, Steelman LS, Shelton JG, Blalock WL, Franklin RA and McCubrey JA: Involvement of PI3K/AKT pathway in cell cycle progression, apoptosis, and neoplastic transformation: a target for cancer chemotherapy. Leukemia 17: 590-603, 2003.

9. Datta SR, Brunet A and Geenberg ME: Cellular survival: a play in three Akts. Genes Dev 13: 2905-2927, 1999.

10. Yang E, Zha J, Jockel J, Boise LH, Thompson CB and Korsmeyer SJ: Bad, a heterodimeric partner for Bcl-XL and Bcl-2, displaces Bax and promotes cell death. Cell 80: 285-291, 1995.

11. Ottilie S, Diaz J-L, Horne W, Chang J, Wang Y, Wilson G, Chang S, Weeks S, Fritz L and Oltersdorf T: Dimerization properties of human $\mathrm{BAD}$. Identification of a BH-3 domain and analysis of its binding to mutant BCL-2 and BCL-XL proteins. J Biol Chem 272: 30866-30872, 1997.

12. Zha J, Harada H, Osipov K, Jockel J, Waksman G and Korsmeyer SJ: $\mathrm{BH} 3$ domain of $\mathrm{BAD}$ is required for heterodimerization with BCL-XL and pro-apoptotic activity. J Biol Chem 272: 24101-24104, 1997.

13. Cardone MH, Roy N, Stennicke HR, Salvasen GS, Franke TF, Stanbridge E, Frisch S and Reed JC: Regulation of cell death protease caspase- 9 by phosphorylation. Science 282: 1318-1321, 1998.

14. Dahia PL, Aguiar RC, Alberta J, Kum JB, Caron S, Sill H, Marsh DJ, Ritz J, Freedman A, Stiles C and Eng C: PTEN is inversely correlated with the cell survival factor Akt/PKB and is inactivated via multiple mechanisms in haematological malignancies. Hum Mol Genet 8: 185-193, 1999.
15. Brazil DP, Park J and Hemmings BA: PKB binding proteins. Getting in on the Akt. Cell 111: 293-303, 2002.

16. Filippa N, Sable CL, Hemmings BA and van Obberghen E: Effect of phosphoinositide-dependent kinase 1 on protein kinase $B$ translocation and its subsequent activation. Mol Cell Biol 20: 7712-7721, 2000.

17. Paramio JM, Segrelles C, Ruiz S and Jorcano JL: Inhibition of protein kinase B (PKB) and PKCzeta mediates keratin K10induced cell cycle arrest. Mol Cell Biol 21: 7449-7459, 2001.

18. Guerra B, Issinger O-G and Wang JYJ: Modulation of human checkpoint kinase Chk1 by the regulatory beta-subunit of protein kinase CK2. Oncogene 22: 4933-4942, 2003.

19. Seeber S, Issinger O-G, Holm T, Kristensen LP and Guerra B: Validation of protein kinase CK2 as oncological target. Apoptosis 10: 875-885, 2005.

20. Guerra B, Siemer S, Boldyreff B and Issinger O-G: Protein kinase CK2: evidence for a protein kinase CK2beta subunit fraction, devoid of the catalytic CK2alpha subunit, in mouse brain and testicles. FEBS Lett 462: 353-357, 1999.

21. Niefind K, Guerra B, Ermakowa I and Issinger O-G: Crystallization and preliminary characterization of crystals of human protein kinase CK2. Acta Crystallogr D 56: 1680-1684, 2000.

22. Boldyreff B, Meggio F, Pinna LA and Issinger O-G: Reconstitution of normal and hyperactivated forms of casein kinase- 2 by variably mutated beta-subunits. Biochemistry 32 : 12672-12677, 1993

23. Rasmussen T, Skjøth IHE, Jensen HH, Niefind K, Boldyreff B and Issinger O-G: Biochemical characterization of the recombinant human Drosophila homologues Timekeeper and Andante involved in the Drosophila circadian oscillator. Mol Cell Biochem 274: 151-161, 2005.

24. Schultz RM, Merriman RL, Andis SL, Bonjouklian R, Grindey GB, Rutherford PG, Gallegos A, Massey K and Powis G: In vitro and in vivo antitumor activity of the phosphatidylinositol-3-kinase inhibitor, wortmannin. Anticancer Res 15: 1135-1139, 1995

25. Davies SP, Reddy H, Caivano M and Cohen P: Specificity and mechanism of action of some commonly used protein kinase inhibitors. Biochem J 351: 95-105, 2000.

26. Lin JM, Kilman VL, Keegan K, Paddock B, Emery-Le M, Rosbach M and Allada R: A role for casein kinase $2 \alpha$ in the Drosophila circadian clock. Nature 420: 816-820, 2002.

27. Channavajhala P and Seldin DC: Functional interaction of protein kinase CK2 and c-Myc in lymphomagenesis. Oncogene 21: $5280-5288,2002$

28. Glass DB, Cheng HC, Mende-Mueller L, Reed J and Walsh DA: Primary structural determinants essential for potent inhibition of cAMP-dependent protein kinase by inhibitory peptides corresponding to the active portion of the heat-stable inhibitor protein. J Biol Chem 264: 8802-8810, 1989

29. Sarbassov DD, Guertin DA, Ali SM and Sabatini DM: Phosphorylation and regulation of AKT/PKB by the Rictor-mTOR complex. Science 307: 1098-1101, 2005.

30. Olsen MEK and Litchfield DW: Order or chaos? An evaluation of the regulation of protein kinase CK2. Biochem Cell Biol 82: 681-693, 2004.

31. Niefind K, Guerra B, Ermakowa I and Issinger O-G: Crystal structure of human protein kinase CK2: insights into basic properties of the CK2 holoenzyme. EMBO J 20: 5320-5331, 2001.

32. Chen M, Li D, Krebs EG and Cooper JA: The casein kinase II beta subunit binds to Mos and inhibits Mos activity. Mol Cell Biol 17: 1904-1912, 1997.

33. Di Maira G, Salvi M, Arrigoni G, Marin O, Sarno S, Brustolon F, Pinna LA and Ruzzene M: Protein kinase CK2 phosphorylates and upregulates AKT/PKB. Cell Death Diff 12: 668-677, 2005.

34. Song G, Ouyang G and Bao S: The activation of AKT/PKB signaling pathway and cell survival. J Cell Mol Med 9: 59-71, 2005.

35. Slaton JW, Unger GM, Sloper DT, Davis AT and Ahmed K: Induction of apoptosis by antisense CK2 in human prostate cancer xenograft model. Mol Cancer Res 2: 712-721, 2004.

36. Wang H, Davis A, Yu S and Ahmed K: Response of cancer cells to molecular interruption of the CK2 signal. Mol Cell Biochem 227: 167-174, 2001 